\title{
Feasibility, benefits and challenges of using telemonitoring for the aging with Developmental Disabilities (DD): An exploratory study
}

\author{
Priya Nambisan, $\mathrm{PhD}^{1}$, Donna Lamkin, $\mathrm{MA}^{2}$, Carrie DeLong, $\mathrm{RN}^{3}$ \\ 1. Assistant Professor | Dept. of Health Informatics \& Administration, College of Health Sciences, University \\ of Wisconsin - Milwaukee, WI 53201-0413 \\ 2. Chief Program Officer | Center for Disability Services, Albany, New York 12208 \\ 3. Assistant Director of Health Care Services, Center for Disability Services | Albany, New York 12208
}

\begin{abstract}
Telemonitoring is being increasingly used to provide services to patients with developmental disabilities in residential community settings. The objective of this study is to assess the feasibility, benefits and challenges of using telemonitoring for aging patients with developmental disabilities. We also assess the benefits and challenges of telemonitoring for the caregivers of these patients. Focus groups and questionnaire-based surveys were used to collect data from patients and caregivers. The study found that telemonitoring was feasible and beneficial for the aging with developmental disabilities, albeit for those who are moderate to high functioning. It was not beneficial or feasible for those with very low functional capabilities. The study found that telemonitoring was beneficial towards providing more independence, more self-confidence in carrying out daily activities, and more knowledge regarding their disease. The study also found that telemonitoring was useful for caregivers to better understand their patients and their needs, better coordinate the services delivered, and to enhance the satisfaction of caregiving. The discussions include limitations of using quantitative methods in this type of setting.

Correspondence: nambisap@uwm.edu, Lamkin@cfdsny.org

DOI: 10.5210/ojphi.v6i2.5460

Copyright $@ 2014$ the author(s)

This is an Open Access article. Authors own copyright of their articles appearing in the Online Journal of Public Health Informatics. Readers may copy articles without permission of the copyright owner(s), as long as the author and OJPHI are acknowledged in the copy and the copy is used for educational, not-for-profit purposes.
\end{abstract}

\section{Introduction}

It is estimated that there are around 641,000 aging people with developmental disabilities (DD) across the country and this number is set to double by 2030 [1]. Developmental disabilities typically occur during childhood (before the age of 18) and include cerebral palsy, mental retardation, learning disorders, autism, and epilepsy; these conditions have been found to cause significant functional impairment in areas such as independent living, self-care, learning, language skills, and economic self-sufficiency. However, this is not a homogenous population as disabilities vary and the same disability can affect different individuals at varying degrees. As treatments for such childhood diseases are becoming better, people are living longer with such disabilities along with the associated functional impairments. Indeed, a sizable population ends up living to old age. Although this is an improvement in the treatment of DDs, this population 
faces severe disparities in healthcare accessibility [2,3]. There is a higher prevalence of adverse conditions, inadequate and timely attention to healthcare needs [2], lack of health promotion and access to quality healthcare services among this population [3].

One of the main reasons for not getting adequate healthcare, apart from poverty and lack of health insurance, is the functional impairment that is characteristic of this population [4]. Some individuals with DD age prematurely, leading to an onset of age related disorders common in the general population like diabetes and heart disease. In addition, there is also an increased incidence of osteoporosis and dementia with the associated psychiatric problems [5]. All this, along with higher intake of anticonvulsant medications, increases the rate of acute care hospitalizations, emergency care, need for short-term nursing home for rehabilitation and need for community based group home for longer care. Studies have shown that small group settings or group homes that provided these individuals with more focused care and more opportunities for leisure and recreation were better than large facilities for people with developmental disabilities [6,7]. As the life expectancy of this population (currently averaging 66.1) as well as the number of individuals who belong to this vulnerable population both steadily increase, technological innovations would be needed to deliver proper care for this population.

It has been noted in prior studies that adequate and timely preventive care and a multidisciplinary approach to tackle issues for this population would be needed in order to make healthcare provisions both time and cost effective [8-10]. As many of these individuals have communication problems along with many functional disabilities, healthcare workers need to be trained in surveillance methods that are efficient in identifying changing physical needs and use subjective and objective methods to review their medical status. Many researchers and health care providers in this area call for a research agenda that would aggressively examine the relationship between different care models and successful aging in this population [8,11]. As a result, many providers are turning to new technologies such as telemonitoring to provide effective solutions in this regard.

\section{Telemonitoring for the aging with developmental disabilities}

Telemonitoring is defined as "an automated process for the transmission of data on a patient's health status from home to the respective health care setting." [12] Telemonitoring differs from telemedicine in that telemonitoring is limited to support provision for patients who need regular monitoring using various telecommunication technologies, whereas telemedicine is the provision of clinical care in the form of diagnosis, treatment and consultation by a provider using various telecommunication technologies. Telemonitoring allows providers to remotely monitor patient status for long periods of time. For example, a patient diagnosed with heart disease can be monitored for BP at the patient's home. A BP monitor that is connected to a telemonitoring device can be used to record the readings and to transmit them to the healthcare organization.

Telemonitoring has been found to be useful in the treatment of chronic diseases among the general population [12-16], however, there hasn't been any attempt to examine whether such technologies can be effectively used for the chronically ill and aging patients with developmental disabilities [17]. This set of population is unlike any other as the range of disabilities and health issues is quite high in addition to functional impairments and communication difficulties. We predict that the perceivable benefits could be similar to that evidenced in the case of the general population: cost-effectiveness, better health outcomes, reduced hospital utilization, and enhanced caregiver satisfaction. This technology would be particularly useful for the aging with DD 
population due to the following factors: (1) transportation difficulties - more difficult than for general aging population; (2) need for constant and regular monitoring - functional impairments make it difficult for any amount of self care and hence need outside help for regular monitoring; (3) higher need for preventive care - higher number of co-morbidities make it extremely necessary for preventive care as complications are higher than when there is only one chronic disease to deal with; (4) need for cost-effective solutions - the constant attention and care needed for this population can overwhelm any provider economically; (5) lack of understanding of own disease/s - a telemonitoring device that gives immediate feedback might be useful in helping them better understand the associated causal relationships for e.g. high BP would mean you get a call from a caregiver; and (6) need for care in a home setting.

\section{Telemonitoring and caregiver satisfaction}

Despite these varied and important potential benefits, deploying telemonitoring for this particular population can be quite challenging, especially for professional caregivers. First, training them to use the telemonitoring devices could be more difficult compared to the general population. Many patients in this target population may not be able to fully utilize all the features in the telemonitoring device, leading to caregiver frustration and provider dissatisfaction. Lack of understanding on how to use the equipment could create doubts among caregivers as to whether the data they are receiving are reliable enough. In addition, improper use of the telemonitoring equipment could lead to damage of the equipment and financial loss. In this context, the caregivers are not only taking the complete burden of technology adoption on their part they are also expected to understand the challenges of training a set of patients with DD use this technology and adapt themselves. However, once implemented telemonitoring has the potential to reduce time spent with each patient, reduce traveling to each patient location, reduce stress and burnout and overall improve satisfaction with caregiving as caregivers for this population are known to have higher burnout rate and higher level of stress [17] compared to the rest of the population. Hence, the study is also focusing on the benefits, challenges and satisfaction of caregivers when providing care using telemonitoring.

A better understanding of the feasibility and benefits of telemonitoring technology for this population as well as the challenges that the technology may pose for both the patients and their caregivers [18] would provide us with a foundation for further studies in this area. The current study is aimed at developing such an understanding by examining the use of telemonitoring in a healthcare center that primarily provides disability services in the Northeastern region in the US.

\section{Data and Method}

Study subjects: Data was collected from the Center for Disability Services (CFDS) at Albany, NY. We used both quantitative (survey) and qualitative (focus groups) to collect data. The study focused on patients who can be classified as 'aging with developmental disabilities'. Patients who are above the age of 45 were classified as aging given that people with DD age earlier than the general population - and we also did individual evaluation of each case to determine whether a patient can be classified as 'aging with developmental disabilities'. Patients with different levels of functionality were enrolled in the study. The enrollment criteria also included different patient settings where care was provided: Individualized Residential Alternative (IRA); Center Independent Living Facilities, (a set of CFDS supported apartments designated as IRA); and 
private homes. These settings were selected with the objective of representing an array of patient living options in rural, suburban, and urban areas.

A total of 21 patients participated in survey part of the study and a total of 6 patients participated in the focus group. All study subjects had co-morbidities. In addition to DD, these subjects have been diagnosed with one or few of the following diseases - Seizure disorders, Schizophrenia, Parkinson's, physical disabilities, mental retardation, Autism, mental health issues like anxiety disorder and psychoses, hyperlipidemia, gout, poor circulation, insulin-dependent and noninsulin dependent diabetes, hypertension, prostate issues, peripheral vascular disease, recurrent UTIs, severe osteoporosis, recurrent skin breakdown, CP, degenerative joint disease, asthma, peripheral neuropathy, colitis, breast cancer, dyslipidemia, GERD, irritable bowel syndrome, gastritis, hyperlipidemia, Barret's Esophagus, Marfan's syndrome, L ventricular hypertrophy, aortic valve stenosis, arthritis, recurrent asthmatic bronchitis and chronic bronchiectasis. Some subjects have communication difficulties, which is characteristic of DD and the caregiver usually collects medical data from subjective and objective observation. Table 1 provides more details on the patient sample. The patients enrolled in this study who had some level of functional independence were trained to use the telemonitoring device (e.g. for measuring blood pressure, pulse, weight, glucose level etc.). Patients with limited functional independence were assisted by professional caregivers from CFDS.

Table 1: Description of the Patient Sample

\begin{tabular}{|lll|}
\hline Characteristic & n & \% \\
\hline Sex & 11 & $52 \%$ \\
\hline - Male & 10 & $48 \%$ \\
\hline - Female & & \\
\hline Age & 1 & $4.7 \%$ \\
\hline $35-45$ & 6 & $28.6 \%$ \\
\hline $45-55$ & 8 & $38.1 \%$ \\
\hline $55-65$ & 4 & $19 \%$ \\
\hline $65-75$ & 2 & $9.5 \%$ \\
\hline $75-85$ & & \\
\hline Race & 2 & $9.5 \%$ \\
\hline Non Hispanic Black & 19 & $90.5 \%$ \\
\hline Non Hispanic White & \\
\hline & & \\
\hline Geographic Location & & $28.6 \%$ \\
\hline Urban & 6 & $57.1 \%$ \\
\hline Rural & 12 & $14.3 \%$ \\
\hline Suburban & 3 & \\
\hline Living Arrangement & & 0 \\
\hline With Family & 0 & $14.3 \%$ \\
\hline Alone & 3 & 0 \\
\hline With Friend & 0 & $38.1 \%$ \\
\hline Residential Service & 18 \\
\hline Primary Diagnosis & & $4.7 \%$ \\
\hline Mild Intellectual Disability & 8 \\
\hline Moderate Intellectual Disability & 1 & \\
\hline
\end{tabular}




\begin{tabular}{|lll|}
\hline Severe Intellectual Disability & 4 & $19 \%$ \\
\hline Profound Intellectual Disability & 8 & $38.1 \%$ \\
\hline Chronic Disorders & & \\
\hline Psychiatric Disorder & 12 & $57.1 \%$ \\
\hline Seizure Disorder & 2 & $9.5 \%$ \\
\hline Obesity & 2 & $9.5 \%$ \\
\hline Diabetes & 2 & $9.5 \%$ \\
\hline Bowel Dysfunction & 7 & $33.3 \%$ \\
\hline Cardiovascular Disease & 9 & $42.9 \%$ \\
\hline Pulmonary Disease & 5 & $23.8 \%$ \\
\hline Neurological Disorder & 3 & $14.3 \%$ \\
\hline Urological Disorder & 9 & $42.9 \%$ \\
\hline Cerebral Palsy & 9 & $42.9 \%$ \\
\hline Functional Independence & & \\
\hline High & 6 & $28.6 \%$ \\
\hline Moderate & 7 & $33.3 \%$ \\
\hline Low & 7 & $33.3 \%$ \\
\hline
\end{tabular}

We also collected data from caregivers - nurses and care staff at CFDS who used the telemonitoring system or helped patients to use the telemonitoring system. Similar to the patient subjects, we used both quantitative (questionnaire) and qualitative (focus group) methods to collect data from caregiving staff. The objective was to understand the feasibility, benefits and challenges of using telemonitoring system for this subset of population from a caregiver's perspective. While the patients are the end users, for this population group, caregivers play an important role in the continued use of the technology.

Telemonitoring system: The Telemonitoring system included Web-based Genesis DM monitors, card readers and glucose cables from Honeywell HomeMed LifeStream telehealth platform. The Genesis DM unit collects data on BP, Pulse, Oxygen Saturation, weight and glucose. To utilize the Genesis DM for multiple people, an ID card must be used with an ID card reader. Caregivers had access to the Lifestream Platform (GE Honeywell's software program), which records all of the readings and places alerts on results that are out of the parameters designated for each person individually. The clinical staff can then respond to these alerts and then add response notes for that session. The telemonitoring unit also can query the user with regard to subjective data (e.g. is the individual experiencing a headache?), and objective data (e.g., the presence of a bruise). Patients were provided with these telemonitoring devices as well as the training needed to use the devices based on their individual needs. The training was provided by the care staff and a Residence RN at CFDS. Each residential program has an assigned Residence RN for a specific number of hours weekly who is typically responsible for overseeing current health related data (e.g., vital signs, PRN usage, bowel habits, current health concerns, medical or other appointments). This Residence RN was assigned to provide training to the residents themselves when appropriate and/or the caregivers. The training started before the telemonitoring device was implemented and continued through out the time the study was conducted. The Residence RNs are usually on site once weekly, depending on the needs of the patients, and available to care staff during regular hours. A 24/7 on-call RN is available for concerns that arise outside of regular hours. 


\section{Data collection}

We employed both focus groups and questionnaire-based survey to collect data from the study subjects. A total of 21 patients participated in survey part of the study. Questionnaire-based surveys were administered to the study subjects (patients), pre and post intervention. The survey was used to collect data on the quality of life of individual patients, an important health outcome in the use of telemonitoring system. To measure Quality of Life, we used a short 8-item questionnaire adapted from an existing, validated scale [19]. The questionnaire comprised of two sets of questions - the first set had 2 items that asked directly about their quality of life and their satisfaction with their health and the second set had 6 items that were associated with general quality of life from the CDC scale [19]. The two sub-scales were both equal interval Likert type scale and were tested for reliability. Cronbach alpha scores were .828 and .886 respectively. Data on specific health outcomes were taken from individual patient record maintained by the healthcare facility. Pre and post health data was compared (6 months before the start of telemonitoring usage was taken for the pre data and 6 months during the telemonitoring usage was taken as the post data). At the end of the post survey, we also conducted a focus group with 6 patients who were mostly 'moderate to high functioning' (given the fact that they needed the ability to respond to our questions). We also administered questionnaire-based survey to 25 caregivers (associated with the patients in our subject pool), pre and post intervention. The survey was used to collect data on caregiver satisfaction and to evaluate differences in pre and post attitudes towards the new technology. In addition, at the end of the study, we also conducted a focus group with 19 caregivers to collect data on perceived benefits and challenges and feasibility in using the telemonitoring system among the target population.

All the study tools including the survey and the focus group questions were reviewed and approved by the first authors' academic Institutional Review Board (IRB) as well as by the IRB at CFDS.

\section{Focus group method for patients and caregivers}

The focus group was conducted by the first author and it was recorded in writing by the author and a nursing staff from CFDS. The entire focus group was also audio recorded with permission from the participants. The first author, who is not affiliated in anyway with the participants (patients or caregivers), developed the questions, administered and moderated the focus group. The focus group participants for the patient group were recruited based on their functionality and ability to respond to questions and also their interest to participate. All the caregivers were invited to participate in the focus group; 19 out of the 25 subjects participated. There were 2 sessions for the caregivers with 12 participants in the first session and 7 participants in the second session.

We employed the exploratory method for conducting the focus groups. This involved using open-ended questions. This method is deemed appropriate for new areas of study with limited prior theoretical understanding such as the current study context [20].

The focus group study questions for patients related to 1) their change in quality of life, 2) satisfaction with the telemonitoring technology, 3) perceived benefits of the new technology, 4) perceived problems and disadvantages of the technology, 5) whether they will continue using the technology, and 6) suggestions for improvement. The focus group study questions for caregivers were intended to capture 1) caregivers' technology related satisfaction - usability issues, 
convenience and other benefits from the technology itself; 2) caregivers' satisfaction with care provision - self-efficacy, job satisfaction; 3) caregivers' willingness to continue using the telemonitoring device; and 4) other concerns, issues, and suggestions for improvement.

Each of the focus group sessions took about an hour or so.

\section{Data Analysis}

Data collection and analysis for the quantitative part of the study was quite challenging (due to low ' $n$ ') and the results were not conclusive. However, we report that as we intend to discuss the comparative benefits of using qualitative studies for this population subgroup. With the quantitative data from patients, we conducted a paired sample $t$ test to compare pre and post data. The quantitative part with caregivers was also challenging due to the high turnover rate of nursing staff and caregivers.

Data from the focus group were analyzed by a combination of summarizing the discussion and detailed content analysis. The summary of discussion was also partially produced during the focus group discussion by periodically summarizing the groups' answers for each section starting with the prepared open-ended questions and taking their feedback and modifying it until consensus is reached - this is a model out-lined by Richard Krueger [21] for in-session summarizing. The transcript from each focus group was carefully reviewed and detailed content analysis was conducted to breakdown the discussion into individual concepts and then related concepts were grouped together. We also analyzed the most frequently mentioned factors for each focus group discussion section.

\section{Results}

\section{Results from Patient data}

The results with regard to the impact of Telemonitoring on Quality of Life while positive were not conclusive. The mean for Quality of Life was slightly higher for all the indicators in the post data, but not all of them were statistically significant. Two indicators - "To what extent do you feel that physical pain prevents you from doing what you need to do?" and "How healthy is your physical environment?" showed a higher statistically significant mean during the study period (post data) than before telemonitoring usage (pre data). The results for "To what extent do you feel that physical pain prevents you from doing what you need to do?" were the following Mean for pre test was 4.45 with a S.D of .510 and mean for post test was 3.90 with a S.D of .912; $\mathrm{t}(19)=2.604 ; \mathrm{p}<.05)$. The results for "How healthy is your physical environment?" were the following - Mean for pre test was 3.77 with a S.D of .922 and the mean for post test was 4.23 with a S.D of $.752 ; \mathrm{t}(21)=-2.215, \mathrm{p}<.05)$. With regard to the specific health outcomes, each individual's health record was carefully analyzed by an RN for changes in their health conditions, number of hospital/doc visits, emergency room visits and for anything that could be attributed to the daily monitoring using the new technology. We did not find any major changes in health conditions or in number of hospital/doc/emergency room visits and if there were any, it could not be attributed to the use of the telemonitoring system. The relatively short study period (6 months) and the low number of patients in this study might explain the lack of stronger findings with regard to the impact on quality of life. However, the findings from the quantitative analysis were generally positive regarding the impact on health outcomes among the target population. 
The results from the focus group with patients strongly support that telemonitoring is beneficial for aging people with DD. For the open-ended question about quality of life, four patients responded. All the four study subjects said that their quality of life has become better; when asked to elaborate on it, they mostly described how it is benefiting their health - at least two people mentioned that their weight has been going down as a result of the regular monitoring. From the analysis, the terms that did repeat at least 5 times was 'I like it'. However, two people mentioned that 'sometimes it gets on my nerve' which points to some of the usability issues of the system. At least 2 people mentioned the terms 'more independent' and the terms 'doing it myself/on my own'. Two people mentioned that 'it was good because it reminds me to take my medication'. The next section about benefits seemed to resonate the same themes about how it was helping them be aware of their health, better understanding of their disease and the treatments, feelings of safety, security and self-efficacy. In addition, participants also repeated some practical benefits such as self-health monitoring and medication reminders.

Table 2 provides a sample of statements captured from the focus group, which are illustrative of (and provide evidence for) the 'benefits from telemonitoring' and improved 'quality of life'.

Table 2: Focus group statements from Patients

\begin{tabular}{l}
\hline "Very useful to know my BP and weight on a daily basis". \\
"If my weight is going up and my BP reading is not good, I know that I have to eat better \\
tomorrow and exercise". \\
"When I exercise and eat better the reading is better next day, and ever since we got the \\
machine, my weight has been going down and my BP is $120 / 80 "$. \\
\hline "It reminds me of my medications". \\
"It is terrific because I can do it on my own and I can get the values directly". \\
"Before, nobody used to tell me the numbers, they would just say it is not good - now I \\
understand why it is not good". \\
"It energizes me, because I have to get up and go to take the reading, something I look \\
forward to everyday". \\
"I feel safe and secure that the nurses are also getting the readings and I know if \\
something is awfully wrong, I will get a call from them".
\end{tabular}

In summary, the focus group revealed high satisfaction rate and there were no disagreements regarding the benefits or improved quality of life from the use of telemonitoring.

For the open-ended question on issues and challenges of the telemonitoring system, one had difficulty with getting the blood sugar readings, one complained about time settings (that it was different from her home clock), another wished that his doctors would have access to the regular monitoring (he sees a doctor outside of CFDS). For the open-ended question on suggestions for improvement - one said that staff should be able to help more. Other than that there were no suggestions for improvement.

Overall, the focus group results indicate that telemonitoring helps to improve patient literacy/knowledge regarding own disease. This in turn could also explain the increased level of self-confidence and self-efficacy as well as the greater motivation for self-care and prevention. Thus, the study findings (from the survey and the focus group) together indicate a stronger level of satisfaction with the new technology (and the associated benefits) among the higher functioning patients. 


\section{Results from Caregiver data}

On the caregivers' side, we encountered an unanticipated problem - as high turnover rate of the caregiving staff (associated with the patients in our subject pool). This is not unusual in this context as typically there is high turnover rate among nursing staff in these type of contexts $[22,23]$. However, due to this turnover, while we had administered the pre questionnaire for caregivers, the post questionnaire could not be administered as only one staff remained from the original study group. Thus, here, we report only the results from the focus group that we conducted for the caregivers. The questions for the focus groups were similar to that of the patients' focus groups -- their satisfaction with the new technology, perceived benefits of the new technology, perceived problems and disadvantages of the technology and suggestions for any improvement. The caregiver focus group revealed a strong support for the main study theses regarding the benefits from using the Telemonitoring technology among aging patients with DD.

The main benefit from regular telemonitoring from the caregivers' perspective was getting daily values on their patient. Caregivers found that this provided them with better understanding of the patient and more control on what is going on. Caregivers also noticed that some of their patients had a better understanding of their disease and were asking more questions regarding their disease. Many commented on how happy the patients were when they started using it and how they seemed to be looking forward to using the telemonitoring device. Everyone agreed that the above 3 factors improved caregivers' relationship with patients. Table 3 provides a sample of statements from the caregivers in the focus group, which are illustrative of the benefits of telemonitoring.

Table 3: Focus group statements from Caregivers

"Very beneficial to get daily values instead of once a week".

Provides better control and an understanding of what is going on - "I am more aware of the patient".

Provides better understanding of what is a normal value for that patient - "I know what is normal for a patient".

"This method was less intrusive as the patients did this, on their own, privately".

When asked about their concerns with the new technology, the caregivers in the focus group noted that the main concern was that this technology was not quite useful for patients with very low functionality. They did not have any doubts on whether this technology would benefit their higher functioning patients. There were issues associated with specific physical disabilities such as spasticity (which is common among patient with cerebral palsy), where the BP readings were inaccurate due to their movements during blood pressure checks and the staff had to manually administer it. The other main usability issue was associated with the card reader - in some facilities it worked fine, whereas in others, it didn't.

Another issue or concern was associated with the questions posed by the telemonitor with regard to the mental health of the patient. Some thought that the 'yes' or 'no' questions did not capture the actual mental health state of the patients. For example, a question on 'are you feeling happy today' is very difficult to answer in just 'yes' or 'no'. One caregiver found that some patients were not answering these questions truthfully - "I have a patient who says she answers all the questions positively as she did not want anyone to find out that she is not in a good mental health state and when I asked her why she is doing that, she said it was because she didn't want to get anymore drugs" 
When asked about any technology-related improvements they would like to see, some caregivers suggested that the technology should enable adding weight scales for weighing patients in wheelchairs. Some caregivers suggested that they were helping patients use the telemonitoring device but that they themselves did not have access to the recordings (i.e. the data). Typically, the data is sent to the facility where the medical staff has the primary access to the data. The caregivers said that it would be highly beneficial if they also got access to the data.

Overall, the caregiver focus group indicated a unanimous and strong support that the Telemonitoring technology is useful and allowed more independent living for higher functioning patients, but that it was not that much useful for low functioning patients. This conclusion in turn reinforces the findings from the patient survey and focus group and in turn enhances the confidence associated with the support found for our broader study theses regarding the benefit and feasibility of employing telemonitoring technology among aging patients with DD. Next, we discuss the limitation and then implications of these findings.

\section{Limitations}

As mentioned before the low number of subjects was one of the major limitations on the quantitative part of this study. The other limitation is the short duration of the study. It is important to conduct long term studies to better understand the feasibility and benefits of telemonitoring for aging people with developmental studies. Another limitation is that we were able to conduct only 1 session of patient focus group. More participants and more sessions would help in gathering more richer and wider range of information. However, this is an exploratory research and this study may have produced results sufficiently indicative of the benefits of using telemonitoring for this population group.

\section{Discussion \& Implications}

The study's main finding is that it is feasible and beneficial to use telemonitoring for this set of population. At the same time, there are some challenges that we will need to address as practical measures for enhancing the effective use of this technology.

The first challenge relates to the nature of the patients - aging people with DD. These patients require much more training and continued evaluation compared to regular population, however, this study has proven that it is feasible to get them to use a telemonitoring device. Further, the caregivers for this population have a higher level of work stress and burnout than that for the general population [17]. There could also be staff and other workers who are undertrained or under educated, as shortage of caregiving staff for this population is higher than for the rest of the population [22,23]. Hence, training and continued evaluation during the course of the study is highly essential. In addition, studies show that providing care staff with better support and internal locus of control would reduce burnout rate [17,24]. This study showed that the caregivers became more in touch with their patients and expressed more satisfaction with their jobs. Hence, future studies could also focus on whether using telemonitoring system would improve job satisfaction and there by enhance the retention rate of caregiving staff.

Another important implication of the current study is with regard to the need to employ multiple research methods. Some of the research methods typically used in studies focused on the general population are not likely to be appropriate for the focus population (aging people with DD). A combination of varied qualitative and quantitative methods would likely be the best approach 
especially when using telemonitoring for a new set of population group. As our study and another study [25] in this area indicate quantitative methods may not always provide conclusive evidence regarding various health outcomes. However, the focus group from the current study indicates that future studies should focus on outcomes such as feeling of independence, empowerment, better understanding of their own disease and feelings of security. It may also indicate the need for future quantitative studies to narrow the focus to study the impact of telemonitoring in a specific disease context i.e. telemonitoring for developmentally disabled aging population with diabetes. Furthermore, we may also need longitudinal cohort group studies to understand the cost effectiveness, hospital utilization and health outcomes of using telemonitoring. Such longitudinal studies may offer insights that are not derived from the crosssectional studies that are typically done in this context.

There also needs to be more studies to understand the unique needs of this population subset and design more robust telemonitoring systems that include features that can measure the BP of patients with spasticity or design a peripheral scale that can interface with telemonitoring systems that will allow patients in wheel chairs to take their weight. Other features such as wearable monitors that can automatically detect vitals as part of the telemonitoring system can be useful for patients with very low functionality.

\section{Funding Acknowledgement:}

This study was partly funded by the NYS Developmental Disabilities Planning Council.

\section{References:}

1. Heller T, Factor A. Older adults with Developmental Disabilities and their Aging family caregivers. Chicago Research and Training Center on Aging with Developmental Disabilities, University of Illinois at Chicago 2004.

2. Krahn GL, Hammond L, Turner A. 2006. A cascade of disparities: health and healthcare access for people with intellectual disabilities. Ment Retard Dev Disabil Res Rev. 12, 70-82. PubMed http://dx.doi.org/10.1002/mrdd.20098

3. Smith DL. 2008. Disparities in healthcare access for older adults with disabilities. Topics in Geriatric Rehabilitation. 24(3), 192-203. http://dx.doi.org/10.1097/01.TGR.0000333753.90550.7b

4. Neufeld S, Lysack C. 2006. Investigating differences among older adults' access to specialized rehabilitation services. $J$ Aging Health. 18, 584-603. PubMed http://dx.doi.org/10.1177/0898264306291105

5. McCarron M, McCallion P. Cost and quality in residential care for persons with intellectual disabilities and dementia - Final Report. Dublin: Trinity College Dublin 2009.

6. Lakin KC, Anderson DJ, Hill BK, et al. 1991. Programs and services received by older persons with mental retardation. Ment Retard. 29, 65-74. PubMed

7. Wilkinson H, Janicki M. 2002. The Edinburgh Principles. J Intellect Disabil Res. 46, 27984. PubMed http://dx.doi.org/10.1046/j.1365-2788.2002.00393.x 
8. Janicki MP, Dalton AJ, Henderson CM, Davidson PW. 1999. Mortality and morbidity among older adults with intellectual disability: health services considerations. Disabil Rehabil. 21(5-6), 284-94. PubMed http://dx.doi.org/10.1080/096382899297710

9. Griswold KS, Msall ME, Cooke RE. 1987. A university-based health maintenance organization for persons with developmental disabilities. Ment Retard. 25, 223-25. PubMed

10. Forgas JP, ed. Feeling and Thinking: Affective influences on social cognition. New York: Cambridge University Press; 2000.

11. Bigby C. 2002. Ageing people with a lifelong disability: Challenges for the aged care and disability sectors. J Intellect Dev Disabil. 27(4), 231-41. http://dx.doi.org/10.1080/1366825021000029294

12. Paré G, Jaana M, Sicotte C. 2007. Systematic review of home Telemonitoring for chronic diseases: The evidence base. J Am Med Inform Assoc. 14(3), 269-77. PubMed http://dx.doi.org/10.1197/jamia.M2270

13. Robinson S, Stroetmann K, Stroetmann V. 2004. Tele-homecare for chronically-ill patients: improved outcomes and new developments. J Inf Tech in Hlthcare. 2(4), 251-62.

14. Schofield RS, Kline SE, Schmalfuss CM, et al. 2005. Early outcomes of a care coordinationenhanced telehome care program for elderly veterans with chronic heart failure. Telemed $J E$ Health. 11, 20-27. PubMed http://dx.doi.org/10.1089/tmj.2005.11.20

15. Tsang MW, Mok M, Kam G, et al. 2001. Improvement in diabetes control with a monitoring system based on a hand-held touchscreen electronic diary. J Telemed Telecare. 7, 47-50. $\underline{\text { PubMed http://dx.doi.org/10.1258/1357633011936138 }}$

16. Cordisco ME, Beniaminovitz A, Hammond K, Mancini D. 1999. Use of telemonitoring to decrease the rate of hospitalization in patients with severe congestive heart failure. Am J Cardiol. 84(7), 860-62. PubMed http://dx.doi.org/10.1016/S0002-9149(99)00452-X

17. Ford J, Honnor J. 2000. Job satisfaction of community residential staff serving individuals with severe intellectual disabilities. $J$ Intellect Dev Disabil. 25(4), 343-62. http://dx.doi.org/10.1080/13668250020019610

18. Brewer JL, Taber-Doughty T, Kubik S. 2010. Safety assessment of a home-based telecare system for adults with developmental disabilities in Indiana: a multi-stakeholder perspective. J Telemed Telecare. 16(5), 265-69. PubMed http://dx.doi.org/10.1258/jtt.2010.090902

19. Newschaffer CJ. Validation of Behavioral Risk Factor Surveillance System (BRFSS) HRQOL measures in a statewide sample. Atlanta: U.S. Department of Health and Human Services, Public Health Service, Centers for Disease Control and Prevention, National Center for Chronic Disease Prevention and Health Promotion, 1998

20. Powell RA, Single HM. 1996. Focus Groups. Int J Qual Health Care. 8(5), 499-504. PubMed http://dx.doi.org/10.1093/intqhc/8.5.499

21. Krueger RA. Focus Groups: A Practical Guide for Applied Research, 2nd ed. (Thousand Oaks, CA: Sage, 1994). 
22. Stone RI. Long-Term Care for the Elderly with Disabilities: Current Policy, Emerging Trends, and Implications for the Twenty-First Century, Milbank Reports, 2000. http://www.milbank.org/uploads/documents/0008stone/index.html

23. Parish SL, Lutwick ZE. 2005. A critical analysis of the emerging crisis in long-term care for people with developmental disabilities. Soc Work. 50, 345-54. PubMed http://dx.doi.org/10.1093/sw/50.4.345

24. Gray-Stanley JA, Muramatsu N. 2011. Work stress, burnout, and social and personal resources among direct care workers. Res Dev Disabil. May-Jun. 32(3), 1065-74.

25. Takahashi PY, Pecina JL, Upatising B, Rajeev R, Shah ND, et al. 2012. A Randomized Controlled Trial of Telemonitoring in Older Adults With Multiple Health Issues to Prevent Hospitalizations and Emergency Department Visits. Arch Intern Med. 172(10), 773-79. $\underline{\text { PubMed }}$ 